\title{
Philosophiques
}

\section{Dialogue sur l'infinité et la réalité}

\section{Sam Labson}

Volume 10, numéro 2, octobre 1983

Le marxisme cent ans après Marx

URI : https://id.erudit.org/iderudit/203235ar

DOI : https://doi.org/10.7202/203235ar

Aller au sommaire du numéro

\section{Éditeur(s)}

Société de philosophie du Québec

ISSN

0316-2923 (imprimé)

1492-1391 (numérique)

Découvrir la revue

\section{Citer cet article}

Labson, S. (1983). Dialogue sur l'infinité et la réalité. Philosophiques, 10(2), 377-402. https://doi.org/10.7202/203235ar

\section{Résumé de l'article}

Cet essai cherche à faire de la complémentarité entre énergie-idée, structure et fonction, et autres couples de concepts, la base d'une nouvelle ontologie qui puisse résoudre les conflits entre les pôles de description " mental » et "physique ", entre la vérité mathématique et la vérité empirique et entre la mécanique quantique et la théorie de la relativité comme formes rivales d'explication scientifique. L'auteur y plaide en faveur de la fermeture déductive (finitude) de l'univers à la lumière de la relation logique entre le nombre (les mathématiques) et l'alphabet (le langage). Il y présente la réalité et l'autonomie conceptuelle des idées (auxquelles se réfèrent les symboles) comme une alternative au platonisme et à d'autres idéologies. Il dispose de fausses interprétations, malentendus, et objections au moyen du dialogue.
Ce document est protégé par la loi sur le droit d'auteur. L'utilisation des services d'Érudit (y compris la reproduction) est assujettie à sa politique d'utilisation que vous pouvez consulter en ligne.

https://apropos.erudit.org/fr/usagers/politique-dutilisation/ 


\title{
DIALOGUE SUR L'INFINITÉ ETT LA RÉALITÉ
}

\author{
par Sam Labson
}

\begin{abstract}
RÉSUMÉ. Cet essai cherche à faire de la complémentarité entre énergie-idée, structure et fonction, et autres couples de concepts, la base d'une nouvelle ontologie qui puisse résoudre les conflits entre les pôles de description " mental » et " physique ", entre la vérité mathématique et la vérité empirique et entre la mécanique quantique et la théorie de la relativité comme formes rivales d'explication scientifique. L'auteur y plaide en faveur de la fermeture déductive (finitude) de l'univers à la lumière de la relation logique entre le nombre (les mathématiques) et l'alphabet (le langage). Il y présente la réalité et l'autonomie conceptuelle des idées (auxquelles se réferent les symboles) comme une alternative au platonisme et à d'autres idéologies. Il dispose de fausses interprétations, malentendus, et objections au moyen du dialogue.
\end{abstract}

ABSTRACT. Conjugacy between energy-idea, structure and function, and other concept pairs is made the basis for a new ontology which resolves the conflicts between "mental" and "physical" poles of description, between mathematical and empirical truth, and between quantum mechanics and relativity theory as rival forms of scientific explanation. The deductive closure (finiteness) of the universe is argued for, in light of the logical connection between number (mathematics) and the alphabet (language). The reality and the conceptual autonomy of ideas (the referents of symbols) are presented as an alternative to Platonism and other ideologies. Mistaken interpretations, misunderstandings and objections are handled through the medium of dialogue.

Je veux d'abord vous présenter mon ami, Sam. C'est un gars bizarre mais j'en prends pas mal de lui parce qu'il est amusant et qu'il vous apporte toujours un tas de livres intéressants. On veille, on parle, on discute jusqu'aux petites heures de la nuit mais avec Sam, on ne s'ennuie pas. Je dois dire que je n'ai jamais le dernier mot dans les discussions bien qu'il ne m'ait encore convaincu de quoi que ce soit. Nos conversations sont 
tellement intéressantes que ce serait dommage que d'autres ne puissent pas en profiter. C'est pourquoi je me suis dit : Pourquoi ne pas leur communiquer l'essentiel de l'apport de chacun ? De cette façon, ils pourront juger. Ainsi, un jour Sam entra chez moi en brandissant un manuel d'algèbre. Voici, en autant que je puis m'en souvenir, l'entretien que nous avons eu.

Sam : Supposons que l'on a les trois variables $(a, b, c)$. Combien de combinaisons peut-on en tirer?

Moi : Tiens, j'ai appris cela au collège. Il y avait une formule ayant trait aux transformations et aux factoriels. Je ne me souviens pas exactement de cette formule mais quand on employait les trois variables à la fois on pouvait facilement arriver à trouver six arrangements différents.

Sam : C'est ça - cette formule est $N$ ! qui dans le cas présent signifie 3, ce qui donne $3 \times 2 \times 1$, c'est-à-dire 6 .

Moi : Alors quand on prenait 2 variables à la fois au lieu de les prendre toutes les 3 à la fois et qu'on multipliait simplement 3 par 2 on obtenait six autres combinaisons.

Sam : D'accord. Maintenant on peut aussi en avoir une seule et alors on a $3 \times 1$ ou trois de plus.

Moi : Donc le nombre total de combinaisons serait : $6+6+$ 3 c'est-à-dire 15 en les prenant 3 à la fois, 2 à la fois et 1 à la fois ce qui épuise le sujet, n'est-ce pas?

Sam : Pas tout à fait. Pourrais-tu maintenant me dire combien 26 variables pourraient donner de combinaisons?

Moi : Non, mais je puis te dire comment le trouver. On prendrait 26 , comme point de départ, on aurait donc $26 \times 25 \times$ 24 , ou, pour s'exprimer en symboles, $n(n-1)(n-2) \ldots$ en décroissant jusqu'à $\times 1$, ou selon le nombre que l'on décide de prendre à la fois. Ça me revient maintenant. Ensuite on recommence comme dans l'exemple précédent en arrêtant à $\times 2$ puis à $\times 3$, ainsi de suite.

Sam : Il y a même une formule pour effectuer ces calculs : $\frac{\mathrm{n} !}{\mathrm{n}-\mathrm{r}}$ où $\mathrm{n}>\mathrm{r}$ (voir Richardson, p. 249).

Moi : Pourquoi toutes ces questions? 
Sam : À cause de l'importance du nombre 26.

Moi : L'importance ! Mes enfants pourraient te répondre là-dessus. C'est le nombre de lettres dans l'alphabet romain. Et puis après?

Sam : Tu vas voir. Dis-moi : qu'est-ce que la réalité ?

Moi : (en riant) J'aurais dû me douter que tu voulais parler philosophie : Pourquoi ne l'as-tu pas dit tout de suite au lieu de tourner autour du pot?

Sam : Réponds seulement à ma question.

Moi : Puisque tu insistes, je vais te donner une belle réponse à la William James. Je suis un pluraliste invétéré. Les promenades dans un parc sont des réalités. Les orages électriques sont des réalités. La naissance et la mort sont des réalités. Les atomes et les protons sont des réalités. Les rêves en tant qu'expérience sont des réalités. Que veux-tu de plus ?

Sam : D'accord, ce sont toutes là des réalités et elles sont toutes différentes par surcroît. Mais, en plus d'être des réalités, elles ont autre chose en commun.

Moi : Et qu'est-ce que cela pourrait bien être?

Sam : Elles reposent toutes sur la constante de Planck.

Moi : Tu veux dire, $b$ ?

Sam : C'est exactement ça $-6.63 \times 10-34$ Joule-sec. Mon ami c'est $b$ qui fait tourner le monde.

Moi : Pas vrai ! Si je ne m'abuse, les physiciens prétendent qu'il y a quelque 500 « constituants ultimes du monde », tous différents (Feinberg, p. 43).

Sam : Oui et non. Il y a environ 500 types de particules subatomiques, mais, par définition, une seule d'entre elles peut être ultime. Si ça n'est pas $b$ ça doit nécessairement être autre chose. Cela n'a vraiment pas d'importance que ce soit $b$ ou autre chose mais il y a au moins une autorité qui prétend que «... . nous avons raison de considérer $b$ comme l'atome d'action ». (d'Abro, Vol. II, p. 461), alors qu'une autre dit que l'équation $\mathrm{E}=\mathrm{hv} \mathrm{s}$ » " est avérée la relation fondamentale de toute la 
physique moderne ", nous obligeant, par conséquent, " . . à considérer les quanta d'action $b$ comme les réalités physiques ultimes dont dépend tout notre monde de phénomènes " (Finkelnburg, pp. 44, 205).

Moi : Oui, mais il y a une différence entre une propriété ou état et une action ou fonction.

Sam : D'accord ; donc, si tu veux considérer la fréquence de radiation (v) des quanta d'énergie $b$ comme la chose fondamentale, à ton goût !

Moi : Non, ce n'est pas ce que je veux dire : bien que $\mathrm{E}=\mathrm{hv}$ soit une relation, on ne peut pas réellement considérer $b$ ou même hv comme des entités. Dans l'équation, $b$ n'est qu'une constante de proportionnalité.

Sam : Ça c'est très astucieux mais c'est aussi erroné. En effet, si je tenais ce raisonnement, je serais obligé de dire que la vitesse de la lumière, $c$, est une " constante de proportionnalité " dans la formule d'Einstein $\mathrm{E}=\mathrm{mc}^{2}$ puisque je peux diviser les deux parties de l'équation et obtenir $C=\sqrt{ } \mathrm{E} / \mathrm{m}$. De la même manière, un écolier pourrait apprendre à considérer $\pi$ comme une simple constante de proportionnalité dans l'équation de la surface du cercle. De cette manière la plupart des concepts fondamentaux n'auraient de sens qu'opérationnel et celui-ci serait sans fondement.

Moi : Je comprends. Cependant, il y a un instant tu disais qu'il n'est pas important que $h$ soit fondamental ou non. Pourquoi le défends-tu avec tant de vigueur, alors?

Sam : C'est parce que, à mon avis, $h$ est la pierre d'assise de la nature, sans elle ou à défaut de tout ce qui est susceptible de la remplacer un jour - on ne saurait expliquer les transformations de l'énergie ; sans elle, la relation d'incertitude de Heisenberg, les équations de Schrodinger et tout le reste, disparaissent. Il est notoire qu'on ne peut bâtir un modèle visuel qui corresponde à nos intuitions mathématiques pour ce qui concerne le domaine subatomique (Blanpied, p. 607), mais on peut difficilement nier la réalité des quanta. On ne peut éluder le pouvoir d'une chose telle que le principe d'exclusion de Pauli, par exemple, principe dont on peut déduire la table périodique tout entière 
(Kauzmann, pp. 315-20 ; Levine, pp. 220-23). Si $b$ ne fonctionnait pas ça serait une autre histoire. Mais puisqu'il fonctionne, on doit prendre au sérieux la prétention à la réalité ultime (Levine pp. 244-46; Livesey, p. 219). Et cette prétention transcende l'importance provisionnelle de $h$ dans la physique contemporaine, du moins, aussi longtemps qu'il y a des gens (comme Bohr et Einstein) qui s'engagent à élaborer des théories sur les implications de leurs formules et ne sont pas de ceux qui se contentent de les appliquer avec succès (MacKinnon, p. 390).

Moi : Disons que j'accepte tes prémisses. Tout ce que tu as réussi à faire c'est de rendre compte du domaine physique.

Sam : Mais il y a plus : L'énergie est dynamique, va sans dire. Or le dynamique et le statique sont complémentaires. J'appelle celui-ci idée et je prétends que dans une description complète de la réalité on doit respecter la conjugalité ${ }^{1}$ entre énergie et idée.

Moi : Ça, ça me dépasse. Tu ferais mieux de t'expliquer.

Sam : Avec plaisir. Prends une chose quelconque - de l'eau, tout simplement. Pour deux molécules d'hydrogène, l'eau en renferme toujours une d'oxygène. Les transformations d'énergie qui donnent l'existence à l'eau ou qui la maintiennent en existence sont une chose ; le rapport fixe de $2: 1$ en est une autre. On ne trouve. pas l'un sans l'autre. Les transformations sont l'aspect dynamique de la réalité : la relation numérique entre les éléments constitutifs en est l'aspect statique. Et ce qui rend cette paire inséparable sauf pour analyse logique ou autre, est ce qu'on peut appeler conjugalité.

Moi : Alors, en physique, position et moment ne sont-ils pas conjugués aussi bien en mécanique neutonienne, qu'en mécanique quantique?

Sam : Absolument. Telles, par exemple, sont les racines imaginaires d'une équation algébrique (Richardson, p. 330). Tel

1. Ici l'auteur - à ce qu'il nous semble - a recours à un néologisme. Il emploie le terme "conjugacy" que nous avons rendu par " complémentarité " dans ce contexte. Mais par la suite nous créerons aussi un mot - pour les besoins de la cause - et nous traduirons "conjugacy" par " conjugalité ". Le mot «complémentarité» n'est pas employé dans le sens de Niels Bohr. N.D.T. 
aussi est hv quoique cela ne nous permette pas de parler de $b$ comme d'un simple facteur de proportionnalité.

Moi : $\mathrm{H}_{2} \mathrm{O}$ peut cependant devenir $\mathrm{H}_{2} \mathrm{O}_{2}$ ou quelque autre substance.

Sam : D'accord, mais la relation entre 2 et 1 qui définit l'eau (en partie) est pour ainsi dire éternelle.

Moi : Comme une forme platonicienne?

Sam : Plutôt comme une forme aristotélicienne, laquelle est inhérente à son sujet. Ceci fournit une explication plus correcte de la «conjugalité ».

Moi : Mais à ce compte, les idées seraient réelles.

Sam : (taquin) Oui, aussi réelles que les orages électriques et les promenades dans le parc. Les idées ont une portée ontologique, il le faut puisqu'elles sont reliées à l'énergie.

Moi : Cela fait penser un peu au « monde 3 » de Popper. (Popper, pp. 116, 300).

Sam : C'est ça, sauf que Popper ne peut rien affirmer au sujet de son troisième monde, à part une conviction vague mais ferme qu'il existe. Même Quine, dans sa critique désormais classique de la dichotomie analyse/synthèse admet que les « facteurs mentaux, comportementaux, ou culturels se rapportent à l'analycité« (Quine, p. 36), et partant qu'il n'existe pas de vérité purement formelle (ni de fausseté).

Moi : Je me souviens vaguement avoir entendu un sémanticien émettre un énoncé à cet effet. On pourrait renforcer son assertion en citant la thèse de Church - la calculabilité efficace est en fin de compte (ultimement) une thèse empirique à propos des calculateurs, humains (ou électroniques), et puisque chaque formule (ou théorème) énumérative se base là-dessus il s'ensuit une conséquence : à savoir qu'on ne peut plus considérer les mathématiques comme une suite de tautologies. (De Long, p. 195). Mais aussi bien l'admettre, Sam, tout ce que tu as réussi à faire en amenant le terme "idée " dans la discussion c'est d'avoir élargi légèrement le champ d'investigation depuis la science naturelle jusqu'à la logique symbolique. Si tu te proposes de 
décrire la réalité de façon exhaustive (ce qui, à mon avis, ne peut se faire), tu devras aller beaucoup plus loin que la discussion présente peut le permettre.

Sam : Très bien. Alors parlons du langage. Es-tu prêt à admettre que les mathématiques sont un symbolisme, que le symbolisme envahit le langage et que, par conséquent, les mathématiques font partie du langage ? Nous pourrions exprimer ceci timidement en disant que le domaine des idées mathématiques est un sous-ensemble du domaine des idées en général, en admettant que le langage est le moyen d'expression de toute espèce d'idées. Ca semble tout à fait inoffensif, n'est-ce pas ?

Moi : Oui, ce qui veut dire que comme Socrate, tu dois avoir un tour dans ton sac.

Sam : L'allusion est flatteuse. Maintenant, essayons d'effectuer une " gödelisation » à rebours. Gödel, tu t'en souviens, a assigné un nombre à chaque élément de syntaxe logique (arithmétisation). Nous pouvons faire l'opposé - c'est-à-dire, prendre chaque opération mathématique et, au risque d'y perdre en clarté et en stylistique (ce qui du point de vue qui nous occupe, n'a aucune importance), lui assigner un nom, ou un vocable. «l devient $\mathrm{u}-\mathrm{n},<+>$ devient $\mathrm{p}-1-\mathrm{u}-\mathrm{s}$; ainsi de suite. Ce que démontre cet exercice qu'il est inutile de poursuivre en détail, c'est que toute communication, par exemple, en mathémaiques, en musique ou en quelque système formel que ce soit, doit pouvoir être complètement exprimable en termes discursifs. La série des nombres et l'alphabet seront les moyens employés à cet effet. Et c'est ici qu'il y a un détail intéressant. En sciences, toutes nos idées sont sujettes à erreur. Elles représentent plus ou moins la réalité, selon le résultat de la vérification de nos hypothèses. Mais, en mathématiques, il n'y a pas de coefficient d'erreur. C'est là le grain de sagesse qui se cache derrière la dichotomie analytique/synthétique même si l'ontologie qu'il soustend a été discréditée. Par définition il y a une relation $1-1$ exacte entre certaines idées (ex : le nombre 17) et notre manière de les représenter.

Moi : Ce n'est pas ce que dit la théorie des ensembles.

Sam : Nous ne parlons pas de la théorie des ensembles en ce moment. Les relations $1-1$, par exemple, entre les nombres 
naturels et réels, dont Galilée, tout d'abord, puis Cantor ont parlé, n'on rien à voir avec notre sujet. Si tu veux une analogie, je te propose la distinction ancienne entre le général (ou les universaux) et le particulier. Le nombre 17 en tant qu'abstraction est parfaitement général (bien que ce soit un nombre spécifique, bien sûr) ; le chiffre qui le représente, que ce soit dans le système décimal ou de notation binaire, est spécifique. Pourtant il y a parfaite corrélation entre eux. On ne peut dire la même chose des idées non mathématiques, où la relation aux faits est toujours quelque chose d'imparfait, et se résume toujours à une question de degrés.

Moi : J'admets l'isomorphisme, mais je ne vois pas ou cela nous mène.

Sam : C'est ici qu'entre en ligne de compte mon « interprétation sémiotique de la réalité ». Prends la suite de raisonnements suivante :

1. toutes les idées peuvent être représentées par le langage ;

2. la représentabilité implique la conversion d'un ensemble de symboles en un autre;

3. or, en termes de l'alphabet considéré comme cadre de tous les systèmes de symboles, toute conversion est possible (Théorie de Gödel inversée) ;

4. donc les mathématiques, comme toute sous-culture linguistique, sont complètement représentables en termes discursifs ;

5. et puisque l'alphabet a 26 lettres, le nombre total d'idées représentables ne peut comme nous l'avons indiqué au début de notre entretien, excéder $26 ! \frac{+26 !}{(26-r !)}+$ . . ., puisque $\mathrm{r}$ augmente de 1 à 25 ; les idées mathématiques feront partie de ce tout ;

6. à la lumière de 5 , on doit dire que le nombre d'idées est fini, quoique très grand, bien entendu ;

7. à la lumière de la « conjugalité » entre énergie et idée, la quantité d'énergie dans l'univers est également vaste mais finie. Et ceci concorde avec les lois de la thermodynamique (entropie). Maintenant tu vois pourquoi je t’ai demandé ce qu'était la réalité. 
Moi : Maintenant, je vois pourquoi nous avons commencé par jongler avec l'algèbre. C'est très impressionnant, mais ta démonstration me semble tout à fait absurde.

Sam : Comment ça ?

Moi : D'abord l'alphabet ne se limite pas nécessairement à 26 lettres ; c'est quelque chose de local.

Sam : C'est vrai mais tout alphabet doit se limiter à un nombre quelconque. $27>26$, mais 27 ! + (etc.) demeure quelque chose de fini. Un alphabet infini impliquerait une contradiction dans les termes. Il ne pourrait y avoir de communication sans le recours à un cadre limité. Et cette communication ne pourrait être maintenue (efficacement sans ce cadre.)

Moi : Même si le nombre d'éléments est fini, qu'est-ce qui empêcherait de les employer pour obtenir des séries infinies d'expressions (bien formées) ou de phrases faites de mots ?

Sam : C'est l'entropie, encore une fois. La construction de phrases requiert une transformation d'énergie. La quantité d'énergie disponible est énorme mais non illimitée. Les transformations ont aussi des bornes supérieures et inférieures - la limite inférieure étant $h$, la supérieure, la quantité d'énergie dans le cosmos.

Moi : Cela n'a rien à voir avec le langage en soi. Ça ne se rapporte qu'à la matière, qu'au domaine physique.

Sam : La conjugalité montre que le langage (c.-à-d. les idées) et la matière (l'énergie) sont ontologiquement inséparables, ils forment une unité. Tu as toi-même mentionné la thèse de Church comme preuve qu'il n'y a pas de calculs sans implications ontologiques.

Moi : Mais Cantor a démontré que l'infini actuel doit exister et que, comme l'a prétendu un auteur, le problème philosophique de l'infini a été résolu (Thomson, Vol. IV, p. 190).

Sam : Cantor était un penseur brillant, mais « . . . bien qu'il crût à la réalité objective de ses nombres transfinis . . . il ne croyait pas que les mathématiciens étaient tenus de considérer ou d'accepter de tels arguments... de telles lignes de pensée .. . n'étaient pas essentielles" (Dauben, p. 128). Pour lui 
il suffisait qu'un programme ou une stratégie mathématique soit logique et qu'on puisse l'effectuer de façon déductive. Et, soit dit en passant, la même chose vaut pour les infinitésimaux : " la théorie modèle ne comporte pas d'engagement dans un sens ou dans l'autre sur des questions ontologiques de cette nature: Ce que les mathématiciens cherchent . . . ce n'est pas l'existence matérielle mais le droit d'user ... de preuves " (Davis et Hersh, p. 252).

Moi : Cependant tu ne trouves pas cela adéquat, métaphysiquement parlant.

Sam : Non, ça ne l'est pas : mais ceci n'est pas du ressort du mathématicien.

Moi : Mais si tu as raison, il n'y a plus d'infini, nous l'avons perdu et nous sommes paralysés dans notre poursuite des mathématiques.

Sam : On ne peut perdre ce qu'on n'a jamais eu ! De plus, nous n'enlevons pas aux mathématiques la possibilité qu'elles offrent d'employer certains concepts de façon fonctionnelle, soit en arithmétique, soit en théorie des ensembles, en analyse nonstandard ou en quoi que ce soit d'autre. Ce que nous nions, c'est que l'infinité soit une structure. On peut continuer de constituer des séries en expansion et d'écrire (. . .) à la suite du dernier membre posé ; on peut continuer d'employer la coupure de Dedekind comme "supposition arbitraire" (Whittaker, p. 27) qui permet de distinguer les nombres rationnels des irrationnels dans le continuum; aucun de nos procédés ne change ; seule change notre interprétation de leur importance.

Moi : On pourrait à juste titre t'appeler un finitiste mais non à titre constructif.

Sam : Exactement. Mes propres arguments causent suffisamment de problèmes aux autres sans que j'aille en emprunter à quiconque.

Moi : Il y a encore quelque chose qui m'embête là-dedans. Par exemple, le théorème de Cantor sur les ensembles de puissances - théorème qui veut que le nombre de sous-ensembles d'un ensemble donné est plus grand que l'ensemble original ou $2^{\mathrm{n}}$ 
$>$ n. Cela montre, entre autres choses, qu'il ne peut y avoir de nombre cardinal plus grand. Est-ce que ça ne renverse pas tes prétentions à la finitude?

Sam : Pas du tout, quoique ta question soit pertinente. Supposons que $\mathrm{n}$ représente l'alphabet comme tu sembles le suggérer. Alors $2^{\mathrm{n}} \mathrm{va}$, bien sûr, excéder $\mathrm{n}$ mais, puisque $\mathrm{n}$ est fini, l'argument initial au sujet des permutations est inéluctable, même pour la Mächtigkeit cantorienne. J'aime bien l'idée que la portée de l'emploi de l'infinité en mathématiques est « . . . tout à fáit verbale et négative à l'effet 'qu'une expression quelconque ne s'applique pas » dans un contexte-de-preuve donné (Lazerowitz, p. 234).

Moi : Aristote, bien sûr, a nié un infini actuel mais il a quand même admis la légitmité d'un infini potentiel, pris comme façon de diviser une grandeur (Physique $207^{\mathrm{b}} 10-11$ ), en tant que processus pouvant être poursuivi indéfiniment quoique jamais complété (ibid., 207 8 , 207 ${ }^{\mathrm{b}} 14$ ).

Sam : Dommage qu'Aristote n'ait pas vécu pour rencontrer Boltzmann. Aucun processus spécifique ne peut se poursuivre indéfiniment. Cependant, l'infini potentiel est un moyen légitime pour décrire les permutations et combinaisons d'éléments structurellement discontinus qu'ils soient $b$ ou qu'ils soient des symboles linguistiques. Sur ce point, je suis d'accord avec le Stagirite. $^{2}$

Moi : Il y a autre chose qui m'ennuie. Si ta dérivation en cinq étapes est valable, il n'y a plus de place pour la nouveauté ; l'univers est fermé. Sûrement quand on pense à Darwin et à ses disciples, cette conséquence-là doit faire réfléchir. Pas besoin d'être un partisan de l'émergence pour se rendre compte que l'indétermination de Heisenberg associée à l'apparition statistiquement improbable quoique historiquement décisive de la

2. Pour plus amples suggestions voir Le Corbeiller p. 875 , où l'auteur développe l'élégante théorie d'un " piège mathématique ", une matrice énumérable basée sur la crystallographie qui capte les caractères saillants et inaitérables de l'univers dans un cadre permanent unique. De plus, la disparition de "l'infini actuel " ne doit nullement nous attrister. Comme -Koyré, Butterfield et d'autres l'ont judicieusement remarqué ; le monde tel que le voit l'homme moderne est incomparablement plus vaste que les imaginations les plus extravagantes (ou les plus disciplinées) des théologiens, des scientifiques, des philosophes et poètes éminents des temps pré-modernes ont pu le concevoir. Voir aussi la note 3. 
mutation des organismes, favorisée (retenue) par la sélection naturelle, est fondamentale à l'histoire de la planète.

Sam : Tu as parfaitement raison. Les règles du jeu d'échecs, elles aussi prévoient des coups et des configurations nouvelles psychologiquement imprévues en dépit du fait que ces règles spécifient de façon exhaustive le nombre de stratégies possibles. L'évolution se trouve dans une situation analogue par rapport à la complexité des lois de la nature et des événements qui se produisirent au cours des quelques millièmes de secondes après le «Big Bang ". Aucun intellect fini (sic) ne peut connaître ces choses dans leur totalité, même si tout est déterminé d'avaǹce. De là vient que la " nouveauté » est réelle et qu'elle est une concession faite à notre ignorance insurmontable.

Moi : Mais la relation d'incertitude nous dit que le hasard fait partie de la nature ; qu'en principe, nos explications sont incomplètes. Tu as l'air de te rabattre sur une version rajeunie du démon de Laplace.

Sam : Ton objection mérite une réponse, mais ça sera long. Rappelle-toi ce que nous avons dit plus tôt en parlant de fonction et de structure. Montrer la structure d'une chose c'est faire voir ce qu'elle est, ce que sont ses parties ; décrire sa fonction c'est spécifier comment ces parties sont reliées entre elles ou ce qu'elles font. Un atome, par exemple, a une structure - il est fait d'électrons, de protons et de neutrons ; et il fonctionne comme l'hydrogène, l'oxygène, l'uranium, le plutonium, ou tout autre chose.

Moi : Je suppose que tu vas maintenant me dire que structure et fonction sont des éléments conjugués d'une même réalité.

Sam : C'est exact ! Bien trouvé ! On peut en discuter séparément mais elles forment une unité. Il y a des fonctions qui ne sont reliées à aucune structure - on pense à l'infinité et aussi au zéro.

Moi : Le zéro ? Tu ne vas pas balancer ça aussi !

Sam : Seulement du point de vue de la structure - du point de vue de la fonction, rien de changé. On peut employer le zéro comme moyen heuristique mais c'est tout. Puisque le tandem 
énergie-idée est complémentaire, l'unité ontologique fondamentale est $1 b$. Pour des raisons obvies, on ne peut avoir $0 b$, à moins de vouloir entreprendre une discussion avec. Parménide ou Heidegger au sujet du néant. Je préfere reléguer le zéro au statut de fonction garantissant la continuité entre nombres entiers négatifs et positifs.

Moi : Les Hindous te sauront gré de cela.

Sam : C'est à espérer. Après tout, ils voulaient que le zéro soit «... une notation pour une position manquante » (Boyer, p. 235), c'est tout. Peano lui-même a commencé son axiomatisation de l'arithmétique en employant le nombre un comme postulat de base (Peano, p. 94).

Moi : Ça se comprend. On peut définir «un〉 d'une façon rigoureuse.

Sam : Pas vrai ! Prends l'approche de Russell-Whitehead qui définit le nombre (cardinal) un comme " . . . la classe de toutes les classes d'unités" (Whitehead and Russell, ${ }^{*} 52$, p. 345). Est-ce qu'il n'y a pas là un relent de circularité (cf. Poincaré, p. 458 ; d'Abro, pp. 192-93)?

Moi : Ce n'est qu'une façon de parler. On peut considérer cette définition comme une façon abrégée de décrire la classe de toutes les classes $\mathrm{x}$ telle que quelque $\mathrm{z}$ est un élément de $\mathrm{x}$, et pour tout $\mathrm{y}$, si $\mathrm{y}$ est un élément de $\mathrm{x}, \mathrm{y}=\mathrm{z}$.> Bon, qu'est-ce que tu dis de cela?

Sam : C'est ridicule. Employer des expressions telles que : " quelque $z$ », « élément de $\mathrm{x}$ », et l'idée d'identité entre y et $\mathrm{z}$ équivaut à faire une pétition de principe car cela présuppose la notion même que tu es en train d'essayer d'expliquer. $\mathrm{Ne}$ vaudrait-il pas mieux admettre avec Poincaré qu'on ne peut expliquer le nombre un? Pourquoi vouloir jouer les esprits rigoureux quand on ne réussit qu'à usurper les concepts qui font l'objet même de la discussion et ainsi créer un cercle vicieux ?

Moi : Si on t'écoutait, le 〈un〉 équivaudrait au ‘point) en géométrie.

Sam : C'est bel et bien ça. Il existe une correspondance biunivoque 1-1 entre les soi-disant «n-tuples ordonnées»de 
l'analyse mathématique et les notions fondamentales de la géométrie analytique. Une autre façon, toute simple, d'aborder le sujet serait de dire que l'idée est indéfinissable ; que le nombre un est un aspect de l'idée et donc que le nombre un est indéfinissable.

Moi : Si on adopte cette ligne de pensée, à la longue, tout devient un terme primitif et se trouve dépouillé de sens.

Sam : Au contraire. Mon optique restitue leur sens au concepts en leur donnant la portée ontologique qu'ils méritent. Déjà les pythagoriciens se rendaient compte qu'il y avait quelque chose d'étrange dans la progression de la série des nombres. C'est qu'ils ne savaient pas exprimer ce qu'ils avaient découvert (au sujet des incommensurables).

Moi : Et toi, comment l'exprimerais-tu ?

Sam : Très simplement. Le système des nombres est intégral quant à la structure mais illimité du point de vue de la fonction. Cela veut dire que tandis que les nombres eux-mêmes sont des unités discontinues, les lois qui régissent leurs interrelations sont continues. C'est ce dernier fait qui explique la vérité métaphorique cachée sous la notion d'expansion décimale "sans fin » puisque, comme nous l'avons déjà dit, aucune transformation réelle ne peut se poursuivre indéfiniment.

Moi : Tout ça est beaucoup trop abstrait. Ça ne résout aucun problème concret, même pas une interprétation défendable de quelque théorie obscure.

Sam : Je ne peux laisser passer un tel défi. Songe à Einstein. Il passa toute sa vie à essayer de surmonter la mécanique quantique pour arriver à un compte rendu de la nature qui exclurait le singulier. Il ne s'est pas aperçu qu'il avait la solution devant lui sous forme de conjugalité entre quanta discontinus et relations de (champ) continues. On ne peut trouver les uns sans les autres. Si Bohr ne s'était approprié le terme de ccomplémentarités en parlant d'autre chose, j'en proposerais l'emploi dans le cas présent. Songe à la quête d'Einstein pour une " description complète " de la nature. Il était loin de se douter que les outils pour y arriver étaient là devant ses yeux. Tout ce qui lui manquait pour voir que continuité et discontinuité sont également fondamen- 
tales à la réalité et que l'invariance (de la loi physique) ne peut se passer ni de l'une ni de l'autre, c'était d'ajuster ses lentilles conceptuelles. Il était incapable de ce " gestalt switch », comme dirait Kuhn. (Kuhn, p. 115)

Moi : Est-ce que les physiciens ne sont pas justement en train de se pencher là-dessus ? Est-ce qu'ils n'essaient pas d'unir les petites et grandes forces, soit, électromagnétisme et gravité en une "grande théorie unifiée " (Wilkinson, p. 5) ?

Sam : Oui et Hawking et d'autres ont accompli beaucoup de travail admirable dans ce sens. Mais tout cela est subtilement entaché de la présupposition que la réalité « objective " est physique et que les concepts sont purement « subjectifs ». On dit souvent, par exemple, qu' " en relativité générale, . . . les quatre co-ordonnées ... n'ont plus aucune signification physique immédiate qu'elles ne sont que des symboles mathématiques ... . (Rosenthal-Schneider, p. 139), comme si le fait d'être "seulement" un symbole était quelque chose dont il faille rougir et comme si sa relation à la nature était un accident ou un caprice quelconque.

Moi : Dans ce cas, nous ferions mieux de discuter les «formes symboliques » selon l'optique de Cassirer.

Sam : Une attitude kantienne comme la sienne ne fait que renforcer la dichotomie subjectif/objectif que je déplore. Mon optique tient d'avantage de De Broglie avec ses « ondes de matière » et de Born, qui a été le premier à soutenir que l'onde, ou l'aspect symbolique d'une particule est ce qui guide celle-ci sur sa trajectoire. Tu vas peut-être dire que je suis en train d'essayer de ressusciter certaines de leurs meilleures idées, de leur donner de la dignité, et de les généraliser philosophiquement.

Moi : Mais Sam, tu dois admettre que certaines de tes hypothèses sont plutôt ténues. Par exemple, si $b$ et le principe de conservation de l'énergie sont si fondamentaux alors pourquoi ne pas mesurer l'énergie directement? On ne le peut pas, bien sûr. On ne peut que mesurer les " sauts " et les différences de niveaux d'énergie. Ces intervalles exprimés en termes symboliques, sont purement relatifs, or toi tu les considères comme des absolus. ${ }^{3}$

3. Comme la seconde citation tirée de Wheeler à la page 20 le démontre clairement, nous avons toutes les raisons de considérer les " niveaux " d'existence macroscopiques et micro- 
Sam : La qualité du mouvement est absolue ; sa quantité mesurable ne l'est pas. Qualité et quantité ...

Moi : Tu vas encore me parler de leur conjugalité !

Sam : Précisément. La notion d' « intervalle » est un moyen heuristique - comme tous les moyens de ce genre elle appartient au domaine des idées. Mais tu soulèves là un problème réel ; problème analogue à celui que constituait l'objection de Whitehead aux vues d'Einstein sur l'espace (Singh, p. 302). Il nous faut une unité minimale, $h$ comme étalon pour juger des transformations. Sans une telle uniformité l'univers devient inintelligible. Pourtant on ne peut pas nier que les exposés courants sur le paradigme de la particule élémentaire sont notoirement nébuleux et embrouillés, que ceci est dû, en partie, à l'imprécision philosophique en tant qu'opposée à l'imprécision scientifique (Shrader-Fréchette, p. 434).

Moi : En dépit de ton dernier énoncé, j'éprouve un curieux de sentiment : j'ai l'impression que tu vas te mettre à en remontrer aux physiciens.

Sam : Pas précisément, je vais simplement leur dire comment interpréter. Mais revenons à Einstein. Il se référait constamment à la «Structure espace-temps ", et ne s'apercevait pas, tout ce temps, que l'espace ne peut en aucune façon être considéré comme une structure en tant qu'opposé à une fonction. Ceci n'invalide pas ses équations ; ça ne fait que nous forcer à les réinterpréter.

Moi : Alors, d'après toi, tous les continus, qu'ils soient mathématiques ou autres - jouissent d'une importance égale avec les éléments discontinus qui les composent. Aucun n'est supérieur à l'autre? (De Broglie, p. 221.)

Sam : Il doit en effet en être ainsi. Une fonction est la fonction $d$ une structure, elle établit les lois des transformations qui régissent la structure - et, puisque toutes les fonctions appartiennent au domaine des idées, elles sont, comme elles, mutuel-

scopiqués comme égaux. Pourtant les dimensions et l'âge de l'univers jouissent encore d'un préjugé favorable. C'est qu'ils posent un dilemme conceptuellement plus grand, que l'organisation de l'énergie dans un électron ou une molécule d'ADN. Notre optique élimine cette distorsion. 
lement isomorphes sous leurs aspects les plus cruciaux. Ceci est réellement tout à fait tautologique et partant inoffensif.

Moi : Et puisque les symboles appartiennent au langage; que le langage sert à exprimer des idées et, puisqu'idée et énergie sont nécessairement liées de façon complémentaire, l'idée est forcément réelle?

Sam : Exact. Tu commences à y voir clair. Souviens-toi également qu'aussi longtemps qu'on jongle avec l'« absolu " et le « relatif », le contexte de l'investigation détermine souvent la réponse (provisoire) à ces questions. Par exemple, en biologie, les cellules sont (encore) atomiques tandis qu'en physique ce sont les électrons qui le sont. Une unité structurale dans une discipline peut, sans qu'il y ait contradiction, être considérée comme fonction continue dans une autre. Il y a cependant une base stable sans laquelle toutes ces distinctions seraient dépourvues de sens. L'ignorer ou le nier serait désastreux.

Moi : Je conserve encore des doutes. S'il faut t'en croire, Einstein a fait erreur pour n'avoir pas intégré TRG (la théorie de la relativité générale) à la $\mathrm{MQ}$ (la mécanique quantique) uniquement par aveuglement d'esprit. C'est comme s'il ne voyait pas qu'une telle synthèse puisse rencontrer des difficultés empiriques.

Sam : Des difficultés empiriques, il y en aura toujours. Par exemple, dans un vain effort de compensation pour n'avoir pas su s'attaquer au côté structural de la réalité, on emploie des théories de la supergravitation qui supposent l'existence de gravitons analogues au quanta.

Moi : Un effort vain?

Sam : Oui, en dépit d'espoirs longtemps entretenus, de pouvoir confirmer l'existence d'ondes gravitationnelles, on n'y est pas parvenu (Whittaker, p. 119). On a déduit l'existence d'une particule appelée graviton mais on n'a jamais réussi à l'« isoler " ou à la détecter (Klein, p. 143). Il y a ici un problème plus vaste : celui de l'" observabilité " qui a fait dire à un savant que, " en l'absence de méthodes de détection, les physiciens émettent tout simplement des hypothèses sur les séries de désintégration, et font des conjectures au sujet des propriétés des 
particules élémentaires de courte durée qui en résultent » (Shrader-Fréchette, p. 419). Ces coups en l'air sont tout simplement inutiles.

Moi : Mais comment éviter cela ? Est-ce qu'on ne doit pas pouvoir se fier à des théories vraisemblables, des théories qui réussissent à rendre compte de phénomènes même lorsqu'elles viennent en conflit avec d'autres paradigmes (Hanson, Vol. 7, p. 45)?

Sam : Bien sûr qu'on le doit - mais quand le conflit repose sur une illusion, il faut pouvoir supprimer les obstacles qui empêchent de solutionner les problèmes et éliminer les « crises » proclamées qui troublent les sciences naturelles.

Moi : Mais comment y arriver?

Sam : Dans le cas de gravitons, c'est très simple. Te souvienstu comment Einstein concevait la gravité ?

Moi : Oui, vaguement. Est-ce que, d'après lui elle ne consistait pas, en fait, en la « densité de l'espace dans une région donnée » ou quelque chose du genre ? C'est ce qui lui permit de faire entrer en ligne de compte la géométrie de Riemann et ainsi «... trouver des équations du champ de gravitation en l'absence de matière " (Einstein, p. 143).

Sam : C'est juste ! Maintenant réfléchis un peu aux conséquences de cette définition. Comment l'espace peut-il être « dense » ou comporter une "métrique " dans un milieu vide de matière?

Moi : Je crois que c'est impossible, mais . . .

Sam : Mais Einstein savait ce qu'il disait, d'accord ? Alors, puisque je ne m'immisce pas dans le domaine de la science mais que j'essaie seulement d'attribuer à celle-ci des fondements logiques solides, force m'est de dire qu'il faut réviser l'interprétation de la gravité, y compris les diagrammes de Minkowski, les lignes de l'univers et tout le reste à la faveur d'un nouvel éclairage.

Moi : En quoi cela peut-il bien consister ?

Sam : Prends les points suivants (i) la gravité se mesure au moyen d'un tenseur ; l'inertie, au moyen d'un scalaire. Pourtant, nous 
savons, (d'après le principe d'équivalences), que gravité et inertie sont interchangeables (cf. la fameuse expérience théorique de l'ascenseur telle que décrite dans Einstein et Infeld, p. 226). Puisqu'un tenseur représente la généralisation d'une échelle et puisque " général » et " particulier "sont encore un exemple de conjugalité ontologique, ... ${ }^{4}$

Moi : Je vois où tu veux en venir ; tu veux me dire que la gravité est une idée. Les formes diverses de l'énergie lui sont complémentaires.

Sam : Tu me suis à merveille ! Je peux renforcer cette conclusion d'autres manières. (ii) L'équation atemporelle de Schrödinger nous force à conclure que la notion espace-temps constitue une relation dérivative $: h$, qui joue un rôle fondamental dans cette équation, est une constante dépourvue de dimensions (cf. Finkelnburg, p. 163). Mais la gravité est tenue, avec raison, pour une propriété universelle de la nature. Comment cela se peutil si elle est soumise à la "métricisation " qui en fait quelque chose de dérivatif ? L'unique solution consiste à rejeter l'explication compliquée d'Einstein en faveur de la seule qui soit compatible avec le statut universel de la gravité.

Moi : Mais alors, que deviennent les équations quadridimensionnelles d'Einstein?

Sam : Rien ! On retient les calculs, mais on change la façon de les interpréter. Cependant, ta question est intéressante car il se trouve que (iii) la " mesure » des interactions gravitationnelles donne une puissance relative de $10^{-76} \mathrm{~cm}$ (Gaillard, p. 507) qui est bien en deça du seuil de la dite physicalité. Pas étonnant que la recherche des gravitons ait été infructueuse. Ceux-ci n'existent pas et ne servent à rien comme postulats. ${ }^{5}$

4. La subsistance d'universaux sans particuliers dépend du sort de conditions contraires aux faits, dont la destinée sémantique à son tour dépend du statut accordé à $\emptyset$ comme simple moyen procédural dénué de signification ontologique ; ceci pour des raisons déjà apportées en relation avec le zéro aux pages 12 et 13 .

5. Pourtant une quatrième raison qui nous porte à rejeter les gravitons c'est l'impossibilité (e.g.) de réduire l'attraction de la gravitation terrestre par l'entremêlement de matière supplémentaire. La conclusion inexorable qui découle d'expériences répétées c'est que, parmi les forces universelles, seule la gravité ne peut être abritée (Klein, p. 86). Comment cela, sinon que la gravité est non-dynamique et que partant (encore une fois), elle relève du domaine de l'idée? 
Moi : Alors tu serais d'accord avec les physiciens qui parlent d'ondicules?

Sam : Oui et non. Eux badinent, moi, pas. Ils persistent à voir un clivage entre la réalité physique et la réalité intellectuelle et à exalter la première aux dépens de la dernière. Leurs efforts d'intégration ne sont tous que du placage. Wheeler est la seule grande exception. Lui seul a vu la nécessité d'une "pré-géométrie », et que " . . . la physique doit se bâtir à partir de fondations dépourvues de physique ", où l'on résout le dilemme de l'organisation de ". . . milliards et de milliards d' . . . actes élémentaires de construction" (Wheeler, p. 6). Il ne donne aucune réponse mais il a réussi à faire une percée pour arriver à poser toutes les questions pertinentes. J'ajouterais que le fait de ne pas prendre au sérieux l'ontologie sous-jacente à ces questions ou de ne pas voir au-delà de la sagesse conventionnelle gouvernant la recherche en cours est loin d'être l'apanage d'une seule spécialité. Et il y a aussi des philosophes qui admettent le besoin de reformuler les principes qui gouvernent notre connaissance de l'univers de manière à mettre en évidence la «... relation profonde et intime qui existe entre la structure mathématique d'une théorie, sa structure conceptuelle - nécessaire à la description physique - et son ontologie fondamentale » (Hooker, p. 208 ; cf. ibid., p. 261 pour une comparaison entre des ontologies opposées).

Moi : Et c'est toi qui pourvois à ce besoin de façon plutôt insidieuse?

Sam : Je ne fais qu'attirer l'attention sur ce qui est déjà là. $1 b$ est une structure de base ; les lois de la nature sont des fonctions de base. Le monde s'effondrerait si ces deux choses n'étaient pas mutuellement compatibles. Est-ce que ça peut être plus simple ?

Moi : Mais il y a sûrement une pierre d'achoppement dans le fait de vouloir réconcilier explication statistique et explication causale. Tu escamotes ce point avec une rare aisance.

Sam : Pas du tout. Tu te souviens ? Le langage est fini ; par conséquent, les idées, en tant qu'exprimées par des moyens linguistiques (et il n'y en a pas d'autres) sont nécessairement finies. Puisque énergie-idée forment un tandem inséparable, (les 
niveaux) d'énergie sont nécessairement finis. Ceci concorde avec le principe qui veut que l'énergie ne puisse être créée ni détruite. Tout ce qui arrive repose sur les permutations d'un ensemble strictement limité de relations ordinatrices comprises dans une série compréhensive de postulats de base. C'est là le véritable sens du « déterminisme ». L'explication statistique n'est qu'un type de relation ordinatrice ; ainsi en est-il de la causalité. Il n'y a aucune opposition ou rivalité entre elles (Voir Bunge, pp. $14,26,351$; et les pages 11-12 ci-dessus).

Moi : Alors, un conflit illusoire de plus disparaît quand on en comprend bien les termes.

Sam : Beaucoup de problèmes se trouvent résolus une fois qu'on a adopté ce nouveau cadre d'interprétation.

Moi : Un exemple, s'il te plaît.

Sam : Certaines gens disent que la gravité a une portée infinie (Gaillard, p. 507). Ce sont des paroles en l'air, étant donné la faiblesse de la constante gravitationnelle. Mais, il y a pis. Si la gravité était infinie, elle " dépasserait » les autres forces puisque l'énergie avec laquelle elle est associée augmente en proportion du carré des masses impliquées tandis que (e.g.) l'énergie nucléaire et électromagnétique augmente seulement en proportion de la masse (Klein, pp. 108-09). Le résultat ? L'univers serait un trou noir - c'est dire que nous ne serions pas assis ici à discuter des fondements de la science et des mathématiques. ${ }^{6} \mathrm{Ou}$ bien encore, prends ceux qui entretiennent l'idée oiseuse d'un $b$ qui changerait avec le temps. S'il en était ainsi, $b$ ne serait plus une constante ou, du moins, pas la plus fondamentale. De plus, si $b$ était sans dimensions, une telle supposition ne tiendrait pas. Ou bien encore, considère la prétention, qui a largement cours, selon laquelle le continuum espacetemps peut « . . . avoir des effets physiques" (d'Abro, p. 112) en dépit du fait que ce continuum espace-temps est indépendant de toutes conditions physiques. Comment peut-on soutenir un

6. Voir Singh, p. 203. On y trouve la condamnation de la « conclusion ridicule " concernant le soi-disant infinité de la poussée gravitationnelle. Comme le dit l'auteur, cela entraînetait la réduction de l'univers à un point. Même ceux qui soutiennent que la constante de Hubble sera un jour inversée pour freiner la vitesse de fuite des galaxies et ainsi reconstituer l'univers petit à petit, même ces gens là n'osent aller à cet extrême et avec raison. 
tel paradoxe sinon parce qu'on admet ce que tout non-platonicien répugne à admettre : à savoir que les idées sont réelles, mais que leur réalité est conceptuellement, mais non entitativement distincte de toute autre chose. Comme Russell l'exprime si bien :

La plupart des philosophes ont pensé que le philosophique et le mental ont épuisé à eux seuls, le monde de l'être. Certains ont émis l'opinion que les choses qui font l'objet des mathématiques ne sont évidemment pas subjectives et que, par conséquent, elles sont, de toute nécessité, physiques et empiriques. D'autres ont affirmé qu'elles n'étaient évidemment pas physiques et, que, par conséquent, elles sont nécessairement physiques et mentales. Les deux partis avaient raison quand à ce qu'ils niaient mais ils se trompaient quant à ce qu'ils affirmaient . . .

(Russell, p. 156)

Moi : Alors, selon toi, il n'y a qu'une troisième espèce de réalité, au delà de la polarité de l'esprit et de la matière ?

Sam : Non ! Il n'y a qu'une seule espèce de réalité : la réalité énergie-matière. Mon ontologie est moniste. Certains aspects de cette réalité peuvent être subdivisés (mais seulement pour des raisons linguistiques). Les relations entre les unités fondamentales (1h) sont polarisées, exactement comme les molécules magnétisées qui se mettent en ligne, ou comme l'électron en rotation. Les unités elles-mêmes sont indestructibles, asymétriques (l'énergie est dynamique, tandis que l'idée est statique) et sont des fonctions intégrales.

Moi : On dirait du Spinoza.

Sam : C'est un peu ça. Il y a aussi du Leibnitz là-dedans puisque $1 b$ « actif » correspond de façon assez vague aux monades. Et, bien que l'ontologie de Platon soit incorrecte, il avait raison d'insister sur la réalité des idées, quelles que soient les fausses interprétations qui en ont résulté dans la suite. On ne peut jamais faire fi des intuitions du génie.

Moi : Toi-même tu es peut-être un génie, mais il y a une chose qui t'a échappé.

Sam : Qu'est-ce que c'est ?

Moi : Dans ton désir de vouloir offrir une description complète qui supplanterait Einstein et tous les autres, tu as oublié le 
théorème de Gödel sur l'incomplétude. Tu ne saurais sûrement pas en éviter les implications accablantes.

Sam : Quelles sont ces implications?

Moi : (1) En principe, il y aura toujours des problèmes insolubles - ceci va à l'encontre de certaines de tes principales affirmations.

(2) Puisque cette insolubilité peut être démontrée à priori, tes efforts pour unir les mathématiques et la nature sont voués à l'échec.

Sam : Un instant ! L'« insolubilité » à laquelle tu fais allusion repose sur le célèbre emploi que Gödel fait de la méthode diagonale de Cantor. L' « arithmétisation de la syntaxe » permet simplement à Gödel de substituer des étiquettes numériques (à des formules faites) dans l'arrangement diagonal de Cantor.

Moi : À quoi veux-tu en venir ?

Sam : Rien qu'à ceci : La formule auto-référentielle de Gödel - formule irrésoluble $G$ ( $G$ n'est pas prouvable en $S 〉$ ) est tellement brillante qu'elle a obscurci ce simple fait : la preuve repose sur l'habileté à générer (construire, énumérer de façon récurrente) une telle formule à volonté. Ce n'est pas seulement le caractère d'indécidabilité de $\mathrm{G}$ dans le système $\mathrm{S}$ qui compte mais la possibilité de répéter la même démarche à volonté, de sorte que « . . . tout le processus répété et . . entretenu, un nombre infini de fois . . . [pour] construire un nombre infini de phrases-à-nombre-théorique qui sont toutes vraies mais toutes improuvables ....»(Rogers, p. 203 ; parenthèses ajoutées).

Moi : Ici on entre en conflit avec l'infinité. Ce n'est qu'une fonction, ce n'est pas une structure.

Sam : (Tout excité) Tu as compris ! Une autre manière de s'exprimer serait de dire que puisque $1 h$ est intégral, et que les transformations requièrent de l'énergie, le caractère de réitération perpétuelle de l'indécidabilité gödélienne doit éventuellement s'épuiser. On escamote ce point quand on a allègrement recours à une formule contenant une ellipse ou qu'on emploie des mots à cet effet. ${ }^{7}$

7. Les implications de cette remarque s'étendent à des analyses non-standard (Robinson, pp. 24748), et à des développements post-gödéliens tels que les ensembles créateurs (M. Davis, p. 91). Les infiniment petits et les infiniment grands sont également affectés. 
Moi : Mais, est-ce que tu ne présupposes pas l'existence d'un univers fini et, par conséquent, ne fais-tu pas là une pétition de principe?

Sam : Non. Nous avons déjà établi la finitude sur une base indépendante. Ne te souviens-tu pas de notre discussion de la thermodynamique (Voir plus haut pp. 8-9) ?

Moi : (désespéré) Mais tu n'as tout de même pas encore répondu à ma deuxième objection. Tous tes procédés dialectiques dépendent de la fusion des mathématiques avec la réalité non-mathématique.

Sam : Tu as toi-même répondu à cette objection depuis longtemps.

Moi : (sourcillant) Moi, j’ai fait ça ?

Sam : Pense à ce que tu m'as dit à propos de la thèse de Church (p. 6). Est-ce que cela ne tranche pas la question ? Les mathématiciens, les physiciens, et même les philosophes ont eu en main les bons outils depuis quelque temps déjà. Il ne s'en sont tout simplement pas servis. Il va nous falloir un nouvel appareil mental pour traiter équitablement à la fois la pluralité des choses, et l'unité sous-jacente qui les traverse. Tout ce que je fais c'est de tirer les conséquences logiques des connaissances courantes et de les intégrer de la façon la plus précise et la plus claire possible. Nos divers spécialistes intellectuels manquent de volonté, ou bien, ils sont trop ancrés dans leurs vieilles habitudes pour s'y mettre.

Moi : (excédé) Eh bien! Tu peux toujours réussir à me convaincre - ou à me réfuter - mais il se pourrait que ça ne soit pas aussi facile avec les gros bonnets !

Sam : (innocemment) Je me demande pourquoi. Après tout, le système tout entier est basé sur la compréhension du nombre et de l'alphabet. (souriant) Saisir les liens de conjugalité qui constituent la réalité devrait s'avérer aussi facile que d'apprendre l'a,b,c.

Je ne me souviens plus très bien ce que nous avons dit après cela. Nous avons continué de causer un moment puis Sam s'en est allé prendre son bus pour retourner chez lui. Vous admet- 
trez qu'il est un peu bizarre. Dans mes meilleurs moments, je me prends à penser qu'il est toqué, mais toqué à la manière du renard. Il devrait bien écrire une monographie pour procurer au public la chance de l'entendre. Quand je parle de cela à Sam, il me répond : « Ça se peut - peut-être un jour ». En attendant, jugez vous-mêmes. *

Department of Philosophy, University of San Diego, California 92110

\section{BIBLIOGRAPHIE}

Blanpied, W.A. Physics : Its Structure and Evolution. Waltham, MA, 1969. Boyer, C.B. A History of Mathematics. New York, 1968.

Bunge, M. Causality: The Place of the Causal Principle in Modern Science. Cambridge, MA, 1959.

d'Abro, A. The Rise of the New Physics. 2 Vols. New York, 1951.

Dauben, J.W. Georg Cantor: His Mathematics and Philosophy of the Infinite. Cambridge, MA, 1979.

Davis, M. "Recursive Function Theory." In P. Edwards (ed.), Encyclopedia of Pbilosophy (New York, 1967), Vol. 7, 89-95.

Davis, P.J., and R. Hersh. The Mathematical Experience (introd. G.C. Rota). Boston, 1980.

DeBroglie, L. Matter and Light : The New Physics. Trans. W.H. Johnston. New York, 1939.

DeLong, H. A Profile of Mathematical Logic. Reading, MA, 1970.

Einstein, A. (with H. A. Lorentz, H. Minkowski and H. Weyl). The Principle of Relativity (notes A. Sommerfeld). Trans. W. Perrett and G.B. Jeffery. London, 1923.

Einstein, A., and L. Infeld. The Evolution of Physics. New York, 1938.

Feinberg, G. "Philosophical Implications of Contemporary Particle Physics." In R.G. Colodny (ed.), Paradigms and Paradoxes. Pittsburgh, 1972. 33-46.

Finkelnburg, W. Structure of Matter. 10th éd., trans. O. Matossi-Riechemeier. New York, 1964.

Gaillard, M.K. "Toward a Unified Picture of Elementary Particle Interactions." American Scientist, Vol. 70 (1982), 506 ff.

Hanson, N.R. "Quantum Mechanics, Problems of," in P. Edwards (ed.), Encyclopedia of Philosopby, s.v., Vol. 7, 41-49.

Hooker, C.A. "Metaphysics and Modern Physics." In C.A. Hooker (ed.), Contemporary Research in the Foundations and Philosopby of Qur:ntum Theory. Dordrecht-Holland, 1973.

\footnotetext{
* Je dois des remerciements aux auteurs anonymes d'un grand nombre de commentaires faits sur une première version de ce texte ainsi qu'à la traductrice, Mme Juliette Veilleux, pour son travail soigneux.
} 
Kauzmann, W. Quantum Chemistry. New York, 1957.

Klein, H.A. The New Gravitation : Key to Incredible Energies. Philadelphia, 1971.

Kuhn, T.S. The Structure of Scientific Revolutions, 2nd ed. Chicago, 1970.

Lazerowitz, M. "The Infinite." In G.W. Roberts (ed.), Bertrand Russell Memorial Volume. London and New York, 1979, 222-41.

LeCorbeiller, P. "Crystals and the Future of Physics." In J.R. Newman (ed.), The World of Mathematics (New York, 1956), Vol. 2, 871-81.

Levine, I.N. Quantum Chemistry, 2nd ed. Boston, 1974.

Livesey, D.L. Atomic and Nuclear Physics. Waltham, MA, 1966.

MacKinnon, E. Scientific Explanation and Atomic Pbysics. Chicago, 1982.

Peano, G. "The principles of arithmetic, presented by a new method," trans. J. van Heijenoort. In J. van Heijenoort (ed.), From Frege to Goedel : A Source Book in Mathematical Logic 1879-1931. Cambridge, MA, 1967.

Poincaré, H. "Science and Method," in The Foundations of Science. Trans. G.B. Halsted (introd. J. Royce). Lancaster, PA, 1913.

Popper, K.R. "Epistemology without a Knowing Subject" and "A Realist View of Logic, Physics, and History." Both in Objective Knowledge : an Evolutionary Approach. Oxford, 1972.

Quine, W.V.O. "Two Dogmas of Empiricism." In From a Logical Point of View, 2nd ed. New York, 1961.

Richardson, M- College Algebra, 3rd ed. Englewood Cliffs, NJ, 1966.

Robinson, A. Introduction to Model Theory and the Metamathematics of Algebra. Amsterdam, 1963.

Rogers, R. Matbematical Logic and Formalized Theories. Amsterdam, 1971.

Rosenthal-Schneider, I. "Presuppositions and Anticipations in Einstein's Physics." In P.A. Schilpp (ed.), Albert Einstein : Philosopher-Scientist, 3rd ed. (LaSalle, IL, 1969), Vol. I, 131-46.

Russell, B. Our Knowledge of the External World. New York, 1929.

Shrader-Frechette, K. "Atomism in Crisis : An Analysis of the Current HighEnergy Paradigm." Pbilosopby of Science, Vol. 44 (1977), 409-40.

Singh, J. Great Ideas of Modern Matbematics : Their Nature and Use. New York, 1959.

Thomson, J. "Infinity in Mathematics and Logic." In P. Edwards (ed.), Encyclopedia of Pbilosopby, s.v., Vol. 4, 183-90.

Wheeler, J.A. "Pregeometry : Motivations and Prospects." In A.R. Marlow (ed.), Quantum Theory and Gravitation. New York, 1980, 1-11.

Whitehead, A.N. and B. Russell. Principia Mathematica to *56. Cambridge, 1967.

Whittaker, E. From Euclid to Eddington : A Study of Conceptions of the External World. Cambridge, 1947.

Wilkinson, D. "The Organization of the Universe." In J.H. Mulvey (ed.), The Nature of Matter. Oxford, 1981, 1-28. 